\title{
IL-36 $\alpha$ expression is elevated in ulcerative colitis and promotes colonic inflammation
}

\author{
SE Russell ${ }^{1,2,6}$, RM Horan ${ }^{1,2,6}$, AM Stefanska ${ }^{1,2}$, A Carey $^{2}$, G Leon $^{1,2}$, M Aguilera $^{3}$, D Statovci ${ }^{3}$, \\ T Moran ${ }^{1,2}$, PG Fallon ${ }^{1,2}$, F Shanahan ${ }^{3}$, EK Brint ${ }^{4}, \mathrm{~S} \mathrm{Melgar}^{3}, \mathrm{~S} \mathrm{Hussey}^{2,5}$ and PT Walsh ${ }^{1,2}$
}

A role for the IL-36 family of cytokines has been identified in the pathogenesis of psoriasis. Although significant mechanistic overlap can exist between psoriasis and inflammatory bowel disease (IBD), to date there have been no reports investigating the IL-36 family in gastrointestinal inflammation. Here we demonstrate that expression levels of IL$36 \alpha$ are specifically elevated in the colonic mucosa of ulcerative colitis patients. This elevated expression is mirrored in the inflamed colonic mucosa of mice, wherein IL-36 receptor deficiency confirmed this pathway as a mediator of mucosal inflammation. I/36r - I - mice exhibited reduced disease severity in an acute DSS-induced model of colitis in association with decreased innate inflammatory cell infiltration to the colon lamina propria. Consistent with these data, infection with the enteropathogenic bacteria Citrobacter rodentium, resulted in reduced innate inflammatory cell recruitment and increased bacterial colonization in the colons of $i / 36 r-/$ - mice. I/36r $-/$ - mice also exhibited altered T helper cell responses in this model, with enhanced Th17 and reduced Th1 responses, demonstrating that IL-36R signaling also regulates intestinal mucosal T-cell responses. These data identify a novel role for IL-36 signaling in colonic inflammation and indicate that the IL-36R pathway may represent a novel target for therapeutic intervention in IBD.

\section{INTRODUCTION}

Inflammatory bowel disease (IBD) is a chronic relapsing intestinal inflammatory condition comprising of separate disease manifestations known as ulcerative colitis (UC) and Crohn's disease (CD). ${ }^{1,2}$ Despite significant advances in our understanding of the basic mechanisms that promote these diseases, current treatment strategies usually involve nonspecific suppression of the chronic inflammatory response and are ineffective in a large number of patients. ${ }^{3,4}$ Although the reasons for this are not fully understood, it seems likely to reflect the complex nature of chronic intestinal inflammation. ${ }^{5}$ More recently, it has become apparent that the incidence of early-onset IBD is increasing across the Western society, and it is now estimated that up to a quarter of all cases of adult IBD first arise during childhood or adolescence. ${ }^{6,7}$ However, relatively little is known about the inflammatory mechanisms that promote the earliest stages of the disease. A deeper understanding of how intestinal homeostasis is altered upon disease diagnosis will increase our understanding of how disease develops during its earliest phases, before the chronic inflammatory cascade has taken hold. Such investigations have the potential to reveal new and more specific disease biomarkers, as well as more effective and personalized strategies aimed at treating patients. Added to this, children and adolescents provide a particularly relevant group as they are much less likely to be exposed to confounding variables seen in adult patients, such as cigarette smoking, co-morbidities and prolonged exposure to treatment of both IBD as well as co-morbid conditions.

In an effort to identify novel mediators associated with the pathogenesis of IBD, we have identified an IL-1 family cytokine, IL-36 $\alpha$, as being expressed at significantly higher levels in the colonic mucosa of newly diagnosed and treatment-naive UC patients. The IL-36 family of cytokines are a relatively recently described subset of the larger IL-1 family and are emerging as important mediators of human disease. ${ }^{8}$ The family consists of

${ }^{1}$ Department of Clinical Medicine, School of Medicine, Trinity College Dublin, Dublin, Ireland. National Children's Research Centre, Our Lady's Children's Hospital, Crumlin, Dublin, Ireland. ${ }^{3}$ APC Microbiome Institute, University College Cork, National University of Ireland, Cork, Ireland. ${ }^{4}$ Department of Pathology, University College Cork, Cork, Ireland and ${ }^{5}$ Academic Centre for Paediatric Research, School of Medicine and Medical Science, University College Dublin, Dublin, Ireland. Correspondence: PT Walsh (walshp10@tcd.ie)

${ }^{6}$ These authors contributed equally to this work.

Received 5 May 2015; accepted 15 November 2015; published online 27 January 2016. doi:10.1038/mi.2015.134 
three separate agonistic ligands, designated IL-36 $\alpha$, IL-36 $\beta$, and IL-36 $\gamma$, and a specific IL-36 receptor antagonist (IL-36Ra), all of which act through the IL-36 receptor. Similar to the more extensively characterized 'classical' IL-1 cytokines, IL- $1 \alpha$ and IL-1 $\beta$, IL-36 cytokines are thought to act as important drivers of inflammation, but in a more tissue-restricted manner. ${ }^{9}$ The expression of IL-36 cytokines has been shown to occur at different sites including the lung and skin and can be derived from diverse cell types including keratinocytes, bronchial epithelium as well as macrophages, monocytes and different T-cell subsets. ${ }^{10}$

As with other members of the IL-1 family, IL-36 cytokines also appear to require proteolytic cleavage for full activity. However, the specific proteases involved are yet to be identified. It has been demonstrated that removal of a short domain at the $\mathrm{N}$-terminus is required for the full antagonistic properties of IL-36Ra and can also increase the agonistic properties of all three IL-36 agonist cytokines by up to 10,000 -fold. ${ }^{11}$ In these processed forms, IL-36 agonistic cytokines exert potent proinflammatory effects on both the innate and the adaptive immune systems, promoting dendritic cell activation and the expression proinflammatory mediators such as IL-6, IL-12, and tumor necrosis factor, while also costimulating $\mathrm{CD} 4{ }^{+} \mathrm{T}$ cells to promote T-cell activation and Th1 responses. ${ }^{12,13}$ Conversely, IL-36Ra can specifically inhibit these effects when present in excess quantities. ${ }^{12}$ Together these data, demonstrate that IL-36 family cytokines can function as important mediators at the interface between innate and adaptive immunity and may play a critical role in inflammatory diseases.

A particular focus on the role of IL-36 cytokine family members as orchestrators of inflammatory diseases of the skin has emerged in recent times stemming from the identification of specific mutations in the gene encoding the IL-36 receptor antagonist (IL36RN), which reduces its activity leading to the development of a severe form of psoriasis. ${ }^{14}$ Several elegant studies, using murine models of psoriasis, have confirmed these data and further implicate IL-36 family members as central to disease pathology. ${ }^{15,16}$ Although IL-36 family members have also been implicated as mediators of inflammatory responses in the lung, ${ }^{17,18}$ to date no reports have examined their expression levels in gastrointestinal tissue or whether they play a role in intestinal inflammation or homeostasis.

In this study, we have identified increased levels of expression of IL36A in the colonic mucosa of newly diagnosed pediatric IBD patients. Interestingly, IL36A expression is specifically elevated in UC patients when compared with control patients or those diagnosed with CD. This increased expression is also evident, at the protein level, in the intestinal mucosa of adult UC patients. Increased IL-36 family expression is also observed in the inflamed colonic mucosa in the mouse, where a deficiency in the IL-36R significantly attenuated colonic inflammation confirming the role of this pathway as a mediator of intestinal inflammation. IL-36-receptor deficiency leads to significant levels of protection from disease associated with decreased colonic macrophage and neutrophil infiltration in an acute model of dextran sodium sulfate (DSS)-induced colitis. These effects are mirrored in a Citrobacter rodentium model of infectious colitis, resulting in higher levels of bacterial colonization in the absence of IL-36 signaling. A deeper mechanistic analysis of this model has also revealed an important role of IL-36R signaling in regulating intestinal $\mathrm{T}$ helper cell responses through restricting the development of Th17 responses while promoting Th1 responses. Together, these data identify a novel role for IL-36 $\alpha$ in the pathogenesis of intestinal inflammation in UC.

\section{RESULTS}

Expression of the IL-36 family member IL36A is elevated in treatment-naive ulcerative colitis

To investigate whether IL-36 family member expression is altered in the earliest phases of IBD pathogenesis, we examined levels of gene expression of IL36A, IL36B, IL36G, and the receptor antagonist $I L-36 R N$, in the rectal mucosa of treatment-naive pediatric patients upon first diagnosis with disease (Supplementary Table 1 online). Through this analysis we found that IL36A gene expression was significantly and specifically elevated among UC patients when compared with control patients $(P<0.01)$. Although $I L 36 G$ expression was evident in the rectal mucosa of all patients examined, no differences in its expression levels were found. In contrast, IL36B gene expression was not detected in any tissues, indicating that unlike the skin, this IL-36 family member is not prominently expressed in the human colon. Interestingly, we also found that IL36RN expression levels appear to be decreased specifically among UC patients, although this observation did not reach statistical significance $(P=0.1)$ (Figure 1a). Together these data demonstrate that IL-36 family members are expressed in the human intestine and that IL36A expression is specifically increased in the colonic mucosa of UC patients, upon initial diagnosis.

These data also indicate that IL-36 family members, and IL-36 $\alpha$ in particular, may play an important role in gastrointestinal inflammation. Studies on the role of IL-36 family members as proinflammatory mediators have previously indicated that these cytokines can promote both innate and adaptive immune responses in mice and humans. ${ }^{13,15,19,20}$ Therefore, we also examined in our patient samples the expression levels of genes previously associated with IL-36-dependent signaling. In agreement with previous reports, ${ }^{21,22}$ we found that levels of IL8 (innate) as well as levels of IFNG and IL17A (adaptive) genes were significantly increased among IBD patients when compared with controls (Figure 1b). Although IL-36 family members have not previously been investigated in the context of type 2 immune responses, we also examined the expression levels of cytokines associated with an 'atypical' Th2 response, which has been described in UC. ${ }^{23}$ In agreement with previous reports, ${ }^{24}$ we found that $I L-13$ expression levels were significantly elevated in UC patients, while IL-9 expression was undetectable in any patients examined (Supplementary Figure 1 and data not shown). Although increased IL-36 activity may account, at least in part, for the increased 
a

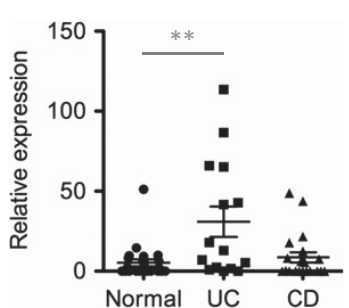

b

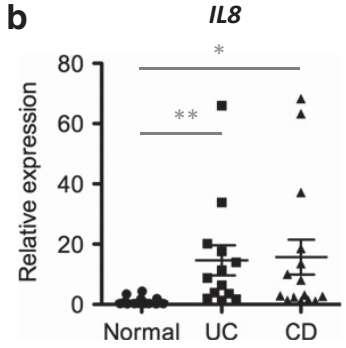

IL36G
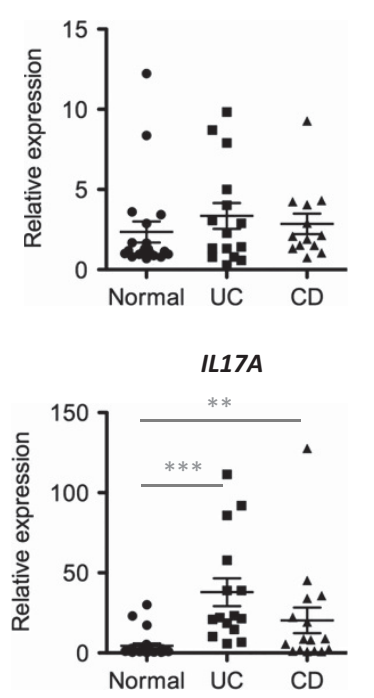
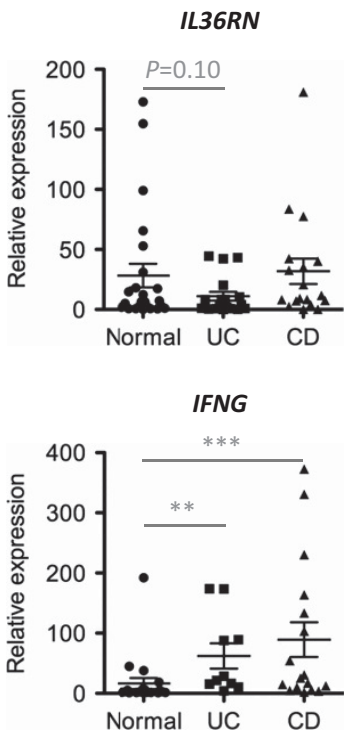

c

Healthy Ctrl

IL-36 $\alpha$

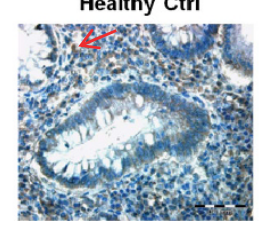

UC

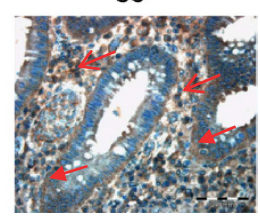

d
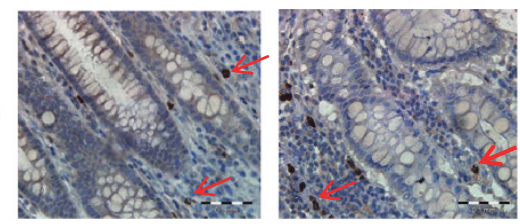

Figure 1 Expression of IL36 $\alpha$ is increased in the colonic mucosa in UC. (a) Relative expression of IL36A, IL36RN, and IL36G mRNA was determined in rectal biopsies from normal controls or treatment-naive pediatric IBD patients by qPCR. Normal $(n=22), \cup C(n=15)$, and CD $(n=18)$. IL36B expression was not detected. (b) Relative expression levels of genes associated with IL-36 mediated innate and adaptive immune responses (IL8, IL17A, and IFNG), determined in rectal biopsies by qPCR. Statistical analysis performed by the Mann-Whitney U-test. ${ }^{*} P \leq 0.05,{ }^{* *} P \leq 0.01$, and ${ }^{* * *} P \leq 0.001$.

Representative analysis of (c), IL-36 $\alpha$ and (d) IL-36R protein expression by immunohistochemistry on colonic tissue sections from adult non-inflamed control and UC patients ( $n=9$ per group). Open arrows denote positive staining on LPMCs. Closed arrows denote positive staining on epithelial cells. CD, Crohn's disease; LPMC, lamina propria mononuclear cell; qPCR, quantitative PCR; UC, ulcerative colitis.

expression of these cytokines in UC, it is clear that other factors are also important in promoting their expression, particularly in the context of CD.

Since IL-1 family member expression and activity are often regulated at the post-transcriptional and -translational level, we next sought to analyze whether IL-36 $\alpha$ expression is altered at the protein level by immunohistochemistry performed on colonic biopsies from an independent cohort of adult UC patients and non-inflamed controls. This analysis confirmed that IL-36 $\alpha$ expression is also elevated at the protein level in UC, with higher expression levels evident among both lamina propria mononuclear cells (LPMCs) as well as epithelial cells (Figure 1c). In contrast, analysis of expression levels of the IL-36R revealed that it is expressed only in a subset of LPMCs and not in colonic epithelium (Figure 1d). Importantly, these data confirm the results of our gene expression analysis, at the level of protein expression, in a separate UC patient cohort, while also revealing information about the cellular targets of IL-36 signaling in the inflamed mucosa.

\section{IL-36R-deficient mice suffer from less severe disease in an innate DSS model of colitis}

As IL-36 $\alpha$ expression is increased in the colons of UC patients, we next sought to investigate what role, if any, this cytokine may play in disease pathogenesis. Firstly, we examined the role of IL-36 family cytokines in an acute innate DSS-driven model of colon injury and inflammation. Importantly, colonic expression of $I L 36 a$ (and $I L 36 g$ ) were also significantly elevated in mice undergoing DSS-induced colitis for 6 days, whereas levels of $I L 36 b$, IL36rn, and the IL36r were not significantly altered, demonstrating that this disease model accurately reflects what we have observed among our patient cohorts (Figure 2a). We examined disease progression in mice deficient in the IL-36 receptor (il36r-/ - ) when compared with WT 

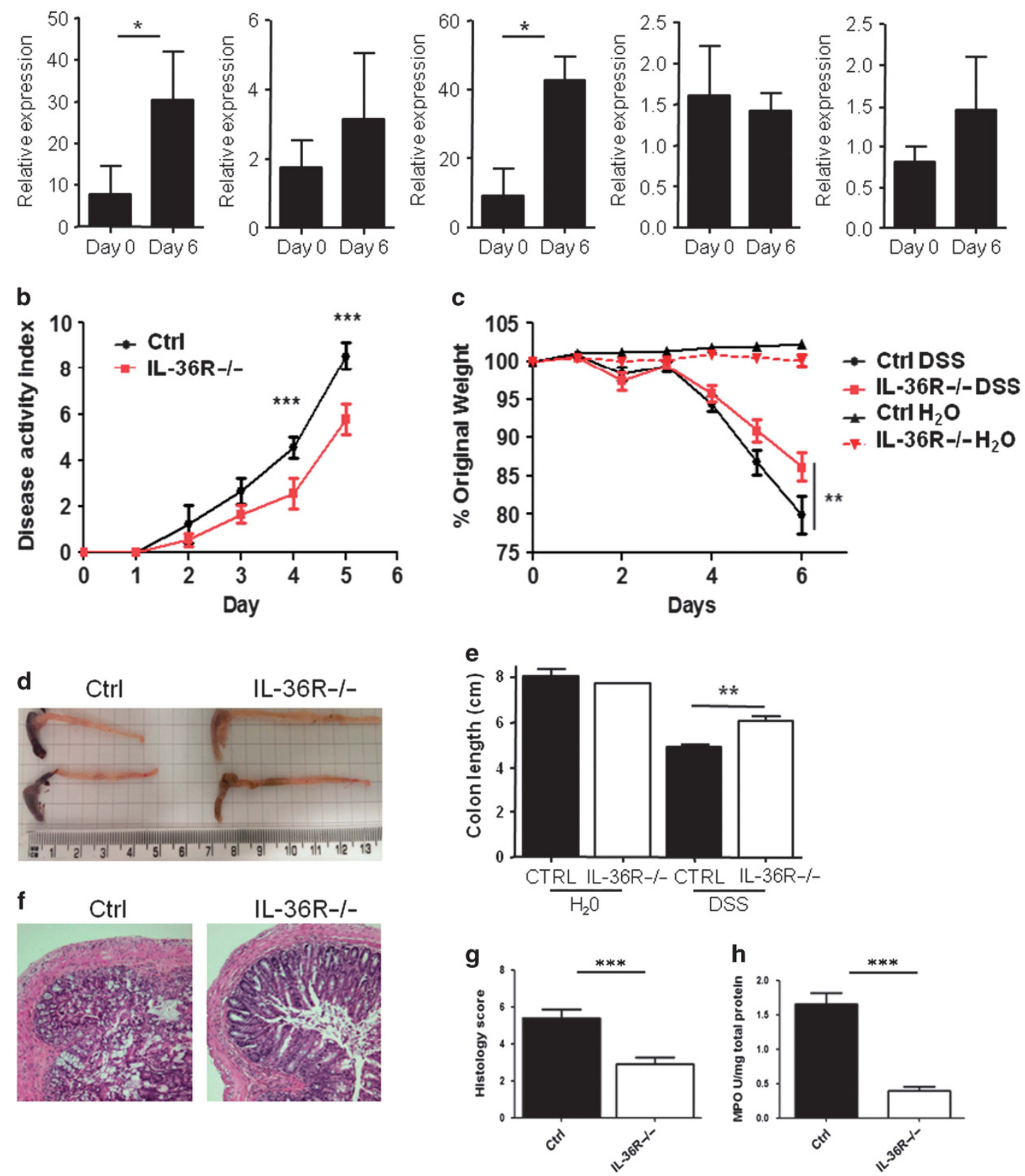

Figure 2 IL-36-receptor-deficient mice suffer from less severe disease in an acute model of DSS-induced colitis. (a) Levels of gene expression of IL-36 family members in colons of WT mice were measured after 1 and 6 days treatment with $2 \%$ DSS by qPCR. WT ctrl and il36r-/ - mice (six per group) were administered 2\% DSS ad libitum in drinking water for 6 days and the development of colitis monitored by (b) disease activity index including fecal occult blood and stool consistency as well as (c) weight loss. After 6 days, the experiment was terminated and (d,e) colon length, (f, $\mathbf{g})$ histology of colon tissues and $(\mathbf{h})$ levels of MPO activity were examined to determine levels of inflammation/tissue destruction. Data representative of four separate DSS experiments (5-7 mice per group) with similar results. Statistical analysis performed by unpaired student $t$-test, ${ }^{\star} P \leq 0.05,{ }^{* \star} P \leq 0.01$, and ${ }^{* \star \star} P \leq 0.001$. DSS, dextran sodium sulfate; MPO, myeloperoxidase; qPCR, quantitative PCR; WT, wild-type.

littermate controls subjected to DSS (2\%) induced acute colitis over 6 days. Here we found that the absence of IL-36R signaling offered significant levels of protection from the disease. This reduced disease in il36r $-/-$ mice was evident throughout the study, in terms of decreased levels of Disease Activity Index (including diarrhea and appearance of fecal blood) and weight loss (Figure $\mathbf{2 b}, \mathbf{c}$ ). Levels of colonic tissue destruction observed were dramatically decreased among il36r - I - mice, when examined at the termination of the study on day 6, through measuring total colon length and histology (Figure 2d-g). The decreased pathology observed, occurred in association with decreased levels of myeloperoxidase (MPO) activity in colon tissue homogenates (Figure 2h). These data confirm that IL-36 family cytokines 

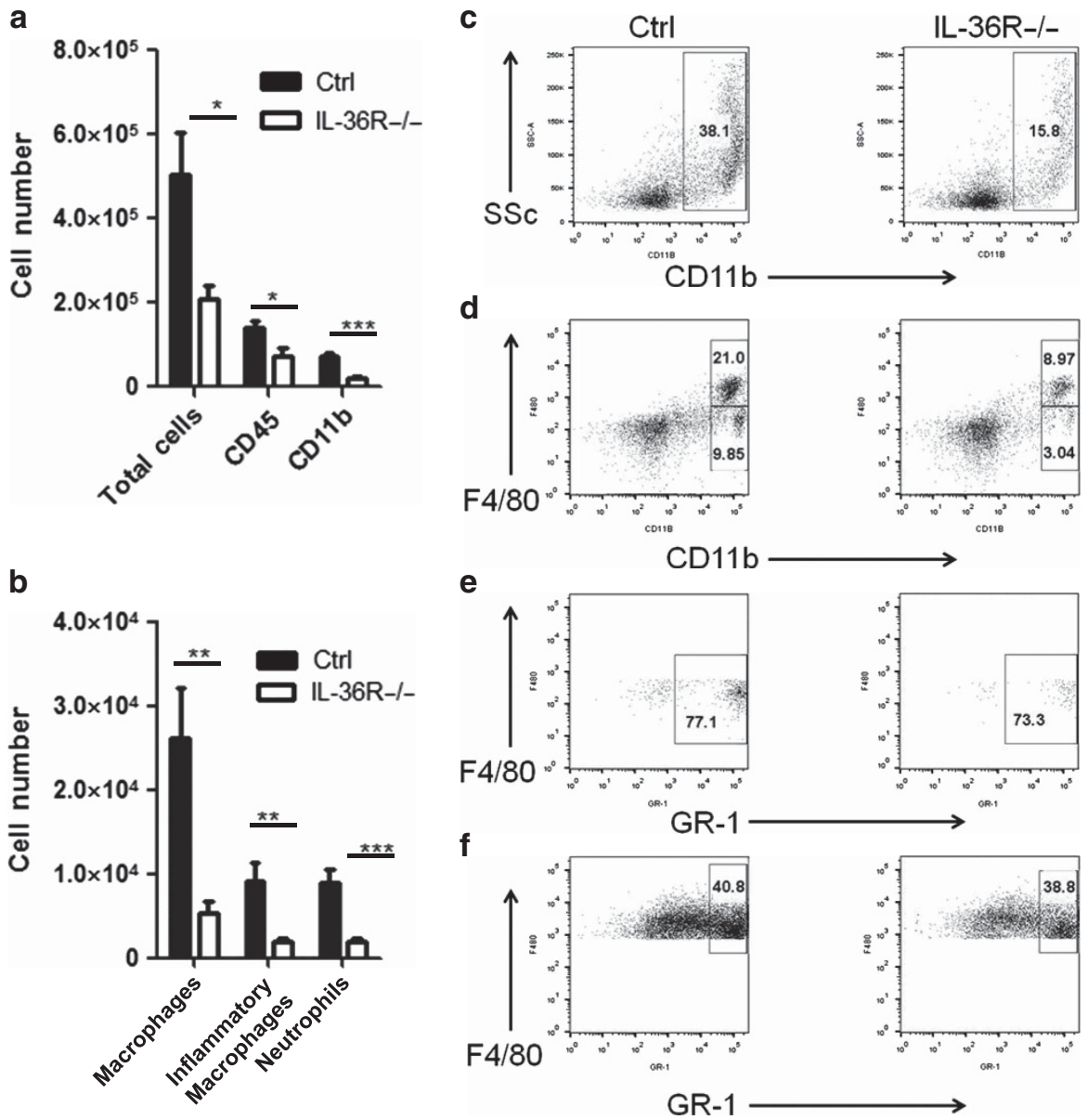

Figure $3 \mathrm{IL}-36$ enhances innate immune cell subset infiltration into the inflamed colons of mice. WT control and il36r- / - mice (3-6 per group) were administered 2\% DSS ad libitum in drinking water for 6 days at which point colons were extracted and examined for levels of innate immune cell subset infiltration into the lamina propria by fluorescence activated cell sorting (FACS). (a) Total live cell numbers as well as CD $45^{+}$and CD $45^{+}$CD11b ${ }^{+}$subsets and (b) $\mathrm{CD}_{4} 5^{+} \mathrm{CD} 11 \mathrm{~b}^{+} \mathrm{F} 480^{+}$(macrophages), $\mathrm{CD} 45^{+} \mathrm{CD} 11 \mathrm{~b}^{+} \mathrm{F} 480^{+} \mathrm{Gr}-1^{+}$(inflammatory macrophages), and $\mathrm{CD} 45^{+} \mathrm{CD} 11 \mathrm{~b}^{+} \mathrm{F} 480^{-} \mathrm{Gr}-1^{+}$ (neutrophils) in the lamina propria of control and $i / 36 r-/-$ mice. Representative FACS analysis showing reduced percentage of (c) total CD11 $\mathrm{b}^{+}$cells and (d) $\mathrm{CD}_{11 \mathrm{~b}}{ }^{+} \mathrm{F}_{480^{+}}$macrophage and $\mathrm{CD} 11 \mathrm{~b}^{+} \mathrm{F}_{480^{-}}$neutrophil subsets as well as (e) unaltered percentages of $\mathrm{Gr}^{+}{ }^{+}$neutrophils and (f) $\mathrm{Gr} 1^{+}$ macrophage subsets between control and $i / 36 r-/$ - mice. Data shown is representative of four independent experiments (3-6 mice per group) with similar results. Data expressed as mean \pm s.e.m. with significance determine by unpaired student's $t$-test. ${ }^{\star} P \leq 0.05,{ }^{* \star} P \leq 0.01$, and ${ }^{\star \star \star} P \leq 0.001$. DSS, dextran sodium sulfate; WT, wild-type.

promote innate intestinal inflammation and play a role in the pathogenesis of colitis.

\section{IL-36 cytokines promote innate inflammatory cell infiltration into the inflamed colon}

In order to investigate the mechanisms through which elevated IL-36 $\alpha$ expression/activity promotes the pathogenesis of UC, we examined the influx of innate inflammatory cell subsets into the lamina propria of WT and il36r - Imice during disease pathogenesis. This analysis demonstrated that the overall numbers of infiltrating cells in the lamina propria, 6 days after the initiation of DSS administration, was significantly reduced in il36r-/ - mice compared with control animals (Figure 3a). This overall decrease in cell numbers was also evident when numbers of total $\mathrm{CD}^{2} 5^{+}$cells, total $\mathrm{CD}_{11 \mathrm{~b}}{ }^{+}$cells, total $\mathrm{CD}_{11 \mathrm{~b}}{ }^{+} \mathrm{F} 480^{+}$ monocyte/macrophages, $\mathrm{CD} 11 \mathrm{~b}^{+} \mathrm{F} 480^{+} \mathrm{GR} 1^{+}$inflammatory macrophages, ${ }^{25,26}$ and $\mathrm{CD}_{11 \mathrm{~b}^{+}} \mathrm{F} 480^{-} \mathrm{Gr}-1^{+}$neutrophils were examined (Figure 3a,b). Interestingly, along with a decrease in overall infiltrating cell numbers, the proportion of total $\mathrm{CD} 11 \mathrm{~b}^{+}$cells, including monocyte/macrophages and neutrophils, expressed as a percentage of the overall CD $45^{+}$ infiltrate, were also decreased in the inflamed colons of $i l 36 r-1-$ mice (Figure 3c,d). Although the relative percentages of $\mathrm{F}_{480} \mathrm{Gr}^{-} 1^{+}$neutrophils (Figure 3e), inflammatory $\mathrm{F}_{480}{ }^{+} \mathrm{Gr}^{+}$monocyte/macrophages (Figure 3f) and $\mathrm{CD} 11 \mathrm{~b}^{+}$Siglec- $\mathrm{F}^{+}$eosinophils (Supplementary Figure 2), were similar between ctrl and il36r-/ - mice, the overall numbers of these subsets were also significantly diminished (Figure $\mathbf{3 b}$ and data not shown). These data indicate that IL-36 cytokines promote colonic inflammation in association with the recruitment of innate inflammatory cell subsets including monocyte/macrophages and granulocytes.

This ability of IL-36 $\alpha$ to promote the infiltration of macrophage and neutrophil subsets in vivo was further confirmed in an intraperitoneal model, whereby 
administration of recombinant IL-36 $\alpha$ led to a significant infiltration of $\mathrm{F}_{480} 0^{\text {intermediate }} \mathrm{Gr}^{+}$inflammatory monocyte/macrophages, ${ }^{27}$ and also neutrophils to the peritoneal cavity within $6 \mathrm{~h}$ (Supplementary Figure 3).

\section{IL-36-deficient mice display altered innate mucosal inflammation and bacterial colonization in Citrobacter rodentium-induced colitis}

Our studies in the DSS model described above clearly demonstrated a role for IL-36R signaling in regulating innate inflammatory responses in the intestine. As IL-36 family members have also been found to regulate $\mathrm{CD} 4^{+} \mathrm{T}$ helper responses in mice, ${ }^{13,28}$ we also explored the role of IL-36R signaling in the $C$. rodentium model of colitis. This model has been extensively used to study mechanisms mucosal inflammation mediated by both innate as well as Th1/Th17-type responses. ${ }^{25,26,29}$

Il36r - / - and il36r + / - heterozygous littermate control mice were orally gavaged with $4 \times 10^{9}$ colony forming units of C. rodentium and disease development was monitored up to 14 days post infection (p.i.). Ctrl and il36r-/ - infected mice presented a similar number of fecal C. rodentium counts from day 2 until day 14 p.i. (data not shown). Bioluminescence imaging analysis of the colons revealed a significantly higher signal in the infected $i l 36 r-1$ - compared with infected ctrl mice at day 7 p.i. (Figure 4a,b), which was still elevated at day 14 (Figure $4 \mathbf{b}, P>0.05$ ), suggesting a deregulated mucosal inflammatory response leading to altered levels of bacterial clearance in the IL-36R-deficient mice. The infection with $C$. rodentium did not spread to other mucosal or systemic organs in either ctrl or il36r - / - mice (Figure 4a). In line with our previous findings, we did not observe major changes in body weight and clinical signs of inflammation in the $C$. rodentium-infected ctrl mice, ${ }^{30}$ and these parameters were at a similar level in the infected il36r - / - mice (data not shown).

Since we identified a reduction in innate immune cell infiltration in the absence of IL-36R signaling in the DSS model, we next asked whether markers associated with neutrophil infiltration are also affected in C. rodentium-infected il36r - / - mice. As expected, levels of $\mathrm{mKC}$ and MPO were significantly elevated in the colons of ctrl mice infected with $C$. rodentium at day 7 p.i. However, these effects were not evident in il36r - / - , indicating that, similar to our data from the DSS model, IL-36R signaling promotes neutrophil infiltration into the colonic mucosa of infected mice (Figure 4c,d).

We also examined inflammatory cell subsets in the mesenteric lymph nodes of infected mice as we have previously described significant alterations in immune cell subset composition at this site in response to $C$. rodentium infection. ${ }^{26,31}$ Although we did not detect any differences in the percentage of total macrophage/monocyte or neutrophil subsets at either timepoint examined p.i. (Figure $4 \mathbf{e}$ and data not shown), we did observe that the percentage of inflammatory $\mathrm{CD} 11 \mathrm{~b}^{+} \mathrm{F} 4 / 80^{+} \mathrm{Gr} 1^{+}$cells were significantly elevated in ctrl mice at day $7 .^{26}$ However, this increase was not seen in the il36r-I- mice (Figure 4e-g). Together these data substantiate our results from the DSS colitis model described above and confirm that IL-36R signaling regulates the recruitment of innate inflammatory cell subsets to the inflamed colons of mice.

\section{IL-36-deficient mice display altered mucosal Th subset responses in Citrobacter rodentium-induced colitis}

Infection with $C$. rodentium results in an induction of an array of T-cell effector responses including Th17 and Th1 cells, which are important for clearance of infection. ${ }^{32}$ To investigate what effects, if any, IL-36 family members might play in mucosal Thelper cell subset responses, we next examined the expression of cytokines and transcription factors associated with these $\mathrm{T}$-cell populations in the colons of both littermate ctrl and $i l 36 r-/-$ mice at day 7 and 14 p.i.. These data are shown as a heat map in Figure 5a. Although, there was no evidence of Th subset responses induced in ctrl mice at the earlier timepoint, we observed that Th17 associated cytokines (Il-17a, Il-23, Il-1 $\beta$, $\mathrm{Il}-6$, and Rorc) were specifically and significantly increased in $C$. rodentium-infected $i l 36 r-1-$ mice at day 7 p.i. relative to expression in infected littermate control mice (Figure 5a,b). The Th1 (Ifng and Tbet) and Treg (Foxp3 and Il-10) associated markers were not significantly induced in infected ctrl or il36r - / - mice at day 7, indicating that IL-36R signaling specifically regulates Th17-type responses at this earlier timepoint (Figure 5a,b).

As expected, by day 14 p.i., Th1 (ifng and tbet) and Treg (foxp3 and il-10) associated markers were clearly significantly induced in infected ctrl mice (Figure 5a). In contrast, there was no significant induction of these responses in the colonic mucosa of $i l 36 r-/$ - mice, even though some markers of Th17 cells (Il-17a and Il-23) remained elevated when compared to ctrl infected mice at this timepoint (Figure 5a,c). Levels of expression of Th2 associated markers (Il-4, Gata3 and Irf4) were low to undetectable in all samples examined (data not shown). In agreement with these gene expression data, we also found $\mathrm{CD} 4^{+} \mathrm{T}$ cells expressing IL-17A to be elevated in the mesenteric lymph nodes of il36r - / - mice when compared with ctrl mice at day 7 p.i. (Figure 5e,f) whereas no induction of Th1 responses was detected in any group examined (Figure 5d). These data demonstrate that as well as regulating innate mucosal responses in the intestine, IL-36R signaling can also regulate the induction of $\mathrm{T}$ helper cell responses to infection with C.rodentium.

\section{IL-36 can directly suppress the generation of Th17 responses while enhancing Th1 responses in vitro}

The data described above demonstrate that IL-36R signaling can regulate intestinal Th cellular subset responses upon infection with C.rodentium. The most striking observations from this data show that the absence of IL-36R signaling results in an elevated Th17-type response earlier during the course of infection without any subsequent induction of the Th1-type response seen in ctrl mice at the later timepoint examined (Figure 5). As we have clearly demonstrated that IL-36R signaling can play a role in regulating innate inflammatory responses, it is possible that 
a
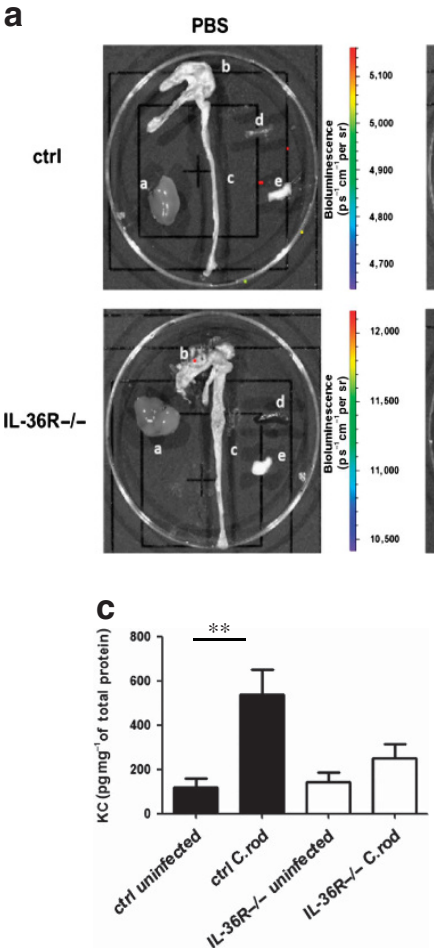

e
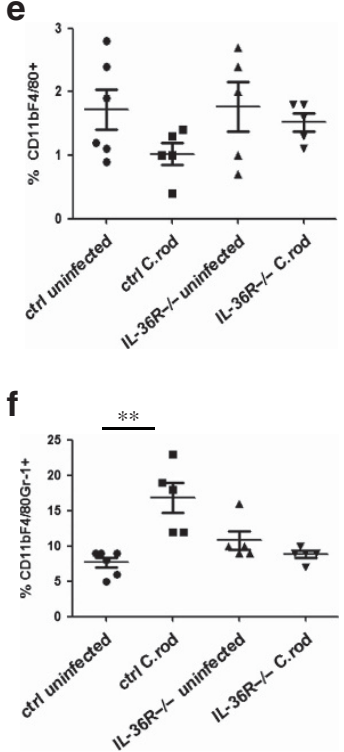

b

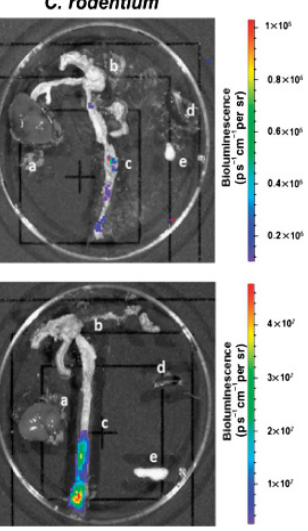

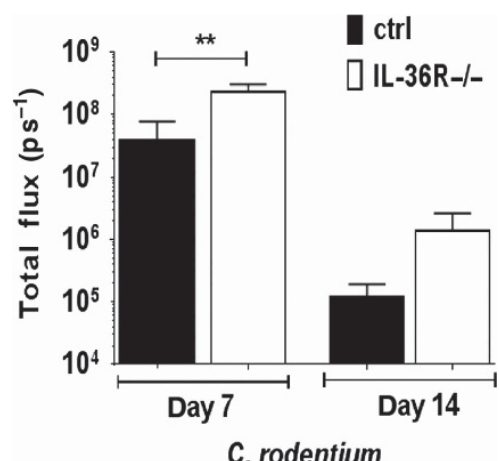

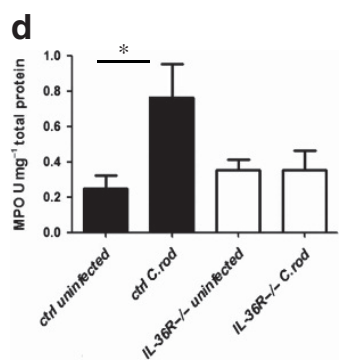

9
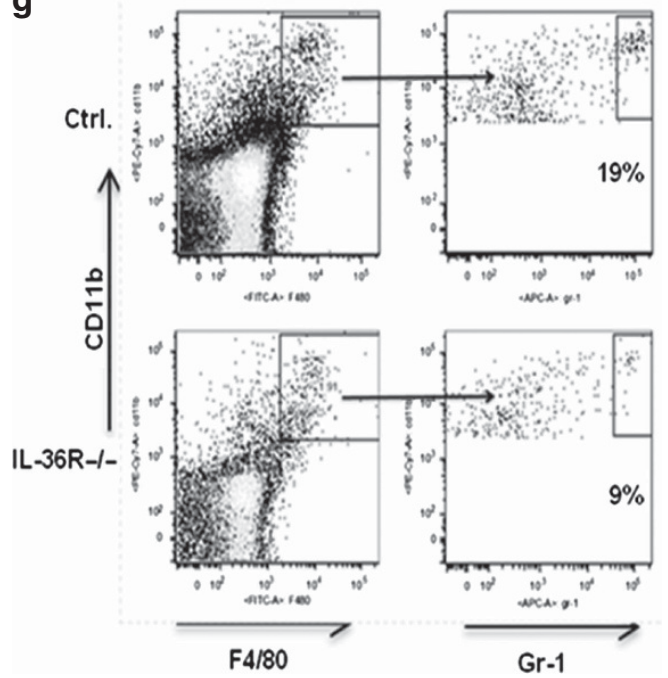

Figure 4 IL-36-deficient mice display altered innate mucosal inflammation and bacterial colonization in C. rodentium-induced colitis. (a) Representative bioluminescence images of (il $36 \mathrm{r}+/-$ ) littermate ctrl and $i / 36 r-I-$ mice at day 7 after PBS treatment of infection with $C$. rodentium obtained using the Xenogen-IVIS Lumina II and showing (a) liver; (b) cecum; (c) colon; (d) spleen; and (e) mesenteric lymph nodes of each experimental group. Color scales represent bioluminescence signal intensity $\left(\mathrm{p} \mathrm{s}^{-1} \mathrm{~cm}^{-2}\right.$ per sr) with red representing the most intense light emission and blue the weakest signal. (b) Colonic bioluminescent signal total flux (p/s) was quantified using the same ROls for all infected animals and including all the distal colons, background was corrected for all animals. Data show total flux $(\mathrm{p} / \mathrm{s})$ on the distal colons of ctrl and $i / 36 r-/-$ infected with $C$. rodentium on days 7 and $14 \mathrm{p.i}$. and is expressed as mean \pm s.e.m. ( $n=5-7$ mice per group). Significance was determined using one-way ANOVA and Bonferroni post hoc ${ }^{* *} P=0.01$. Expression levels of (c) mKC and (d) MPO activity in colonic homogenates of uninfected (PBS) or $C$. rodentium-infected ctrl and il36r- / - mice at day 7 p.i. Protein expression/activity is expressed as $\mathrm{pg} \mathrm{mg}^{-1}$ of total tissue protein from five individual mice per group. Relative percentages of (e) total macrophage $\left(\mathrm{CD} 11 \mathrm{~b}^{+} \mathrm{F} 480^{+}\right)$and (f) inflammatory macrophage $\left(\mathrm{CD} 11 \mathrm{~b}^{+} \mathrm{F} 480^{+} \mathrm{Gr} 1^{+}\right)$subsets in the mesenteric lymph nodes of ctrl and il36r- / infected with $C$. rodentium on day 7 p.i. Each symbol represents values for an individual mouse with five mice per group. (g) Representative fluorescence activated cell sorting showing values from individual mice in (f). Data expressed as mean \pm s.e.m. with significance determined by unpaired student's $t$-test, ${ }^{*} P \leq 0.05$ and ${ }^{* *} P \leq 0.01$. ANOVA, analysis of variance; MPO, myeloperoxidase; PBS, phosphate-buffered saline; p.i., post infection; ROIs, regions of interest. 

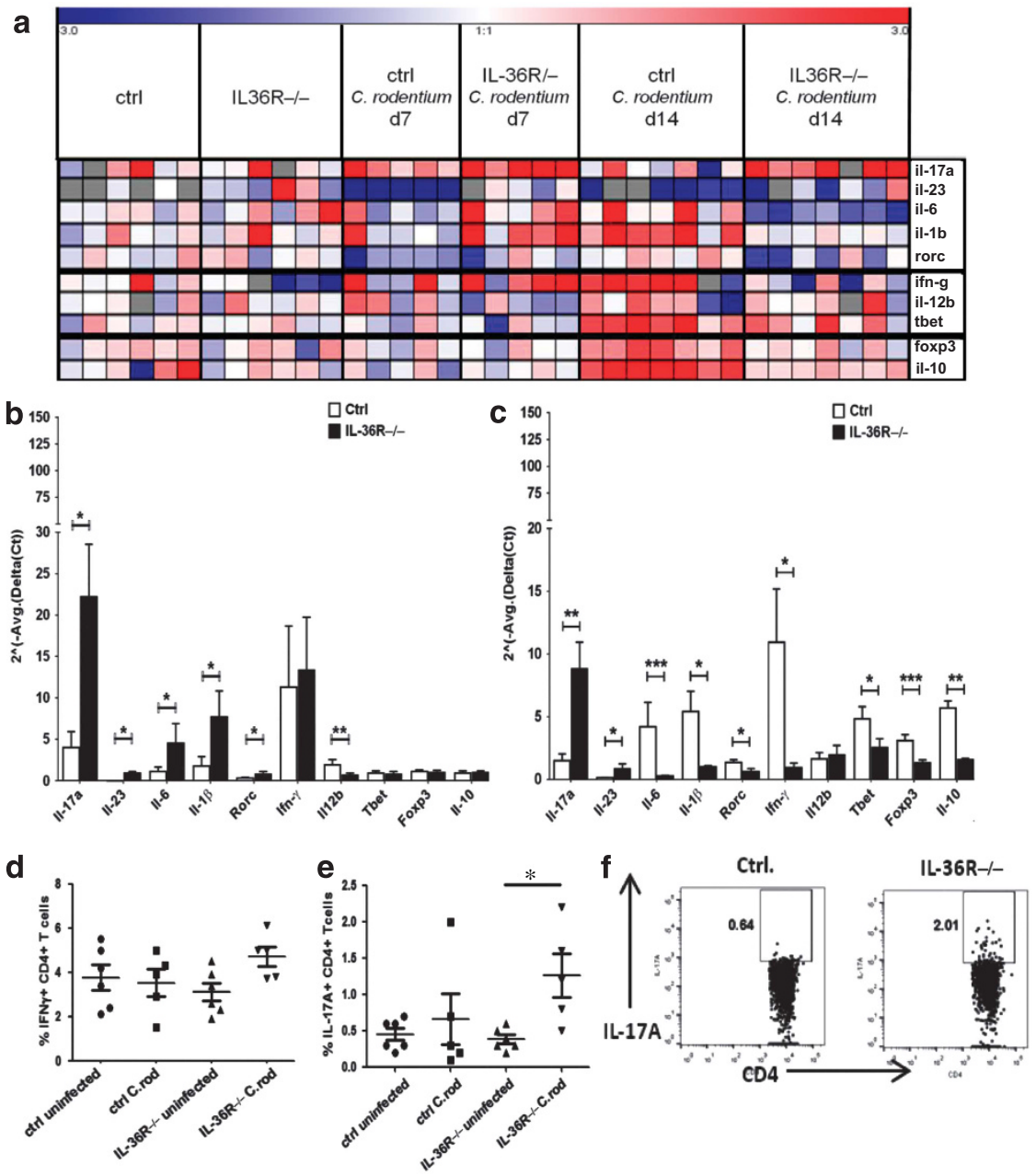

Figure 5 IL-36-deficient mice display altered mucosal Th subset responses in C. rodentium-induced colitis. (a) Relative gene expression heat map from the distal colon mRNA from (il36 + / - ) littermate ctrl and il36r- / - mice analyzed on day 7 and 14 p.i. for the main markers representative of the Th17 (II17a, II23, I/6, IL1b, and Rorgt), Th1 (Ifng, I/12b, and Tbet), and Treg (Foxp3and II10) responses. Color scales show higher relative expression in red and lower relative expression in blue. Each box represents specific gene expression values for individual mice (b,c) Bars represent the mean \pm s.e.m. relative expression of each gene from the colons of 5-7 individual mice at (b) day 7 and (c) day 14 post infection, using the values from uninfected ctrl and il36r $-/$ - mice as their respective baseline. Significance was determined by Mann-Whitney $t$-test ${ }^{*} P<0.05$ and ${ }^{*} P<0.01$. Fluorescence activated cell sorting (FACS) analysis of (d) $\mathrm{CD} 4{ }^{+} \mathrm{IFN} \gamma^{+}$and (e) $\mathrm{CD} 4^{+} \mathrm{IL}-17 \mathrm{~A}^{+}$cells in the mesenteric lymph nodes of uninfected and infected ctrl and $i / 36 r-/-$ mice at day 7 p.i. Each symbol represents values for an individual mouse with five mice per group. (f) Representative FACS showing values from individual mice in e. Data expressed as mean \pm s.e.m. with significance determined by unpaired student's $t$-test, ${ }^{\star} P \leq 0.05$.

the altered $\mathrm{T}$ helper subset responses observed, result from the inability to mount an appropriate innate response to an infection and the subsequently increased bacterial load (Figure 4).

However, several reports have also indicated that IL-36 cytokines can act to directly to regulate $\mathrm{T}$ helper subset and specifically promote Th1-type responses in the mouse. ${ }^{12,13}$ As we have observed a clear pattern of regulation of mucosal $\mathrm{T}$ helper subset responses in this model, we also investigated whether IL-36 $\alpha$ can play a role in modulating T helper cell differentiation in vitro. In agreement with previous reports, we found that the addition of IL-36 $\alpha$ was a potent driver of enhanced Th1 responses (Figure 6a). In contrast, IL-36 $\alpha$ could also directly inhibit the generation of Th17 responses in vitro when analyzed through the induction of $\mathrm{gfp}^{+}$cells using purified $\mathrm{CD}^{+}{ }^{+} \mathrm{T}$ cells from an Il-17a-egfp reporter mouse (Figure 6b).

These data reveal a novel role for IL-36 family cytokines as regulators of inflammatory $\mathrm{T}$ helper cell subset differentiation in vitro as well as in vivo in the C. rodentium infection model.

\section{DISCUSSION}

Despite obvious differences in terms of the predominant tissues affected, it is likely that a degree of mechanistic overlap exists between psoriasis and IBD. This idea is underscored by the discovery that patients with psoriasis exhibit increased rates of IBD, while patients suffering from both $C D$ and UC are also at increased risk of psoriasis. ${ }^{33,34}$ Added to this, the skin is one of the most common extraintestinal tissue systems associated with manifestations of IBD. ${ }^{35}$ In this study, we demonstrate for the 
a

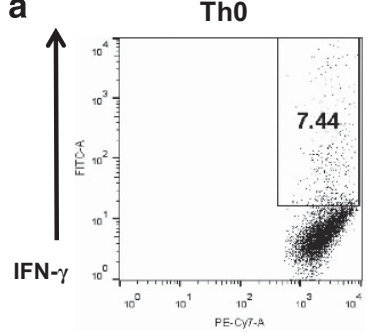

b

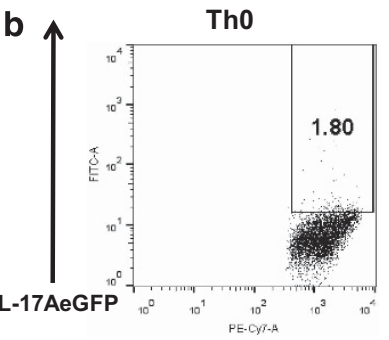

Th 1

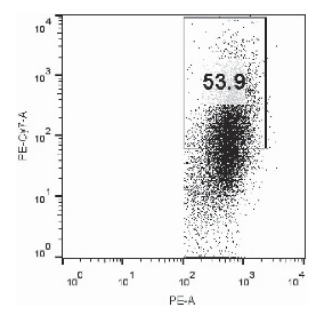

Th 17

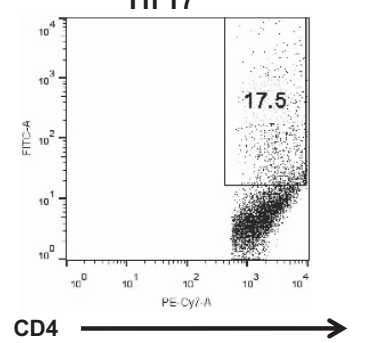

Th $1+$ IL-36

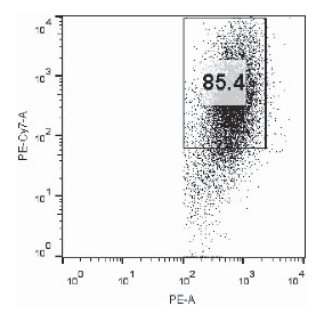

Th $17+$ IL-36

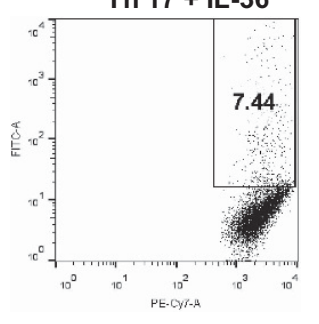

Figure 6 IL-36 can directly suppress the generation of Th17 responses while enhancing Th1 responses in vitro. (a) Magnetic activated cell sorting (MACS)-purified CD4 + T cells from C57BL/6 mice stimulated in vitro under neutral (Th0) or Th1-inducing conditions (IL-12 and anti-IL-4R) in the presence and absence of rIL-36 $\alpha\left(200 \mathrm{ng} \mathrm{ml}^{-1}\right)$ and analyzed for intracellular expression of IFN $\gamma$ by fluorescence activated cell sorting (FACS). (b) MACS-purified CD4 + T cells from C57BL/6 il17a-egfp reporter mice stimulated in vitro under neutral (Th0) or Th17-inducing conditions (TGF $\beta$, IL-6, antiIL-4R, and anti-IFN $\gamma)$ in the presence and absence of rlL-36 $\alpha\left(200 \mathrm{ng} \mathrm{ml}^{-1}\right)$ and analyzed for intracellular expression of green fluorescent protein by FACS. Data shown is representative of three independent experiments with similar results.

first time an important role for IL-36 family cytokines in the pathophysiology of intestinal inflammation in IBD patient samples and mouse models of intestinal inflammation. Although the importance of these cytokines in the context of psoriatic skin inflammation is well established, whether they are important mediators of intestinal homeostasis and inflammation has to our knowledge not been described. In this study, we have identified a specific alteration in the expression levels of IL36A in the colonic mucosa of newly diagnosed and treatment-naive pediatric UC patients. These observations were confirmed at the protein level by IHC of colon tissue biopsies from a separate UC patient cohort. This mirrors what has previously been reported in the lesional skin of psoriasis patients, ${ }^{16}$ indicating this pathway may represent an important mechanistic link between both diseases. Interestingly, although IL36RN expression has been reported to be also elevated in psoriatic lesions, ${ }^{16}$ this is not the case in the intestinal mucosa our pediatric UC patient cohort. Indeed, it appears that IL36RN expression is decreased specifically in the colons of UC patients, although this trend is not statistically significant with the patient numbers included in this study. It will be interesting to determine whether, with an expanded analysis of patients, IL36RN expression is significantly decreased in UC. If so, this would indicate an important dichotomy with the elevated levels of IL36A observed, and would suggest an environment permissive to IL-36R activation specifically in the intestinal mucosa of UC patients.

Elevated IL-36 $\alpha$-protein expression in the colonic mucosa of UC patients appears to localize to both epithelium as well as LPMCs (Figure 1c), indicating that both cell types may be important sources of this cytokine in the inflamed intestine. In contrast, expression of the IL-36 receptor is restricted only to LPMCs (Figure 1d), suggesting that these cells are important mediators of the inflammatory response to elevated IL-36 $\alpha$. In agreement with this data, we have found colonic epithelial cell lines to be relatively insensitive, whereas primary human monocytes respond in a robust fashion to stimulation with IL-36 $\alpha$ in vitro (data not shown).

A further interesting question surrounds whether the altered IL-36 family member expression in UC represents a susceptibility factor for developing disease, or occurs secondary to the local inflammatory response in the colon. In this regard, it is noteworthy that elevated IL-36 $\alpha$ expression was also detected in an adult patient cohort with established disease. In addition, thus far, we have not identified any significant association between IL36A expression levels and the degree of intestinal inflammation in the pediatric patients included in this study (patient details provided in Supplementary Table 1). However, a far greater analysis of patient numbers will be required to specifically address this question. It is interesting to note that in recent times several mutations in the gene encoding IL36RN have been identified as linked to the pathogenesis of generalized pustular psoriasis. To our knowledge, the presence of UC-like manifestations of disease, have not thus far been investigated among these patients. ${ }^{14}$

To determine what role IL-36 family members might play in intestinal inflammation in UC, we investigated the pathogenesis of disease in the il36r-I- mouse using two different mouse models of colitis. Firstly, we examined disease progression of $i l 36 r-/$ - mice in an acute DSS-induced model 
of colon injury and inflammation. This model provides a robust surrogate for human disease, as IL36a (and IL36g) gene expression is also elevated during colitis. Disease pathogenesis in this model is predominantly driven by innate processes of intestinal inflammation, with significant protection from disease observed in the absence of IL-36 signaling, indicating that IL-36 family members promote intestinal immune pathology. Moreover, this protection occurred in association with decreased numbers of infiltrating innate immune cell subsets into the lamina propria of $i l 36 r-/-$ mice, including inflammatory macrophage and granulocyte subsets (neutrophils and eosinophils), which plays important roles in mediating the tissue destruction observed in this model. ${ }^{32} \mathrm{In}$ agreement with these data, we have found that IL-36 $\alpha$ can act as a potent inducer of chemokines associated with neutrophil and macrophage influx, including KC, MIP-2, and MCP-1 from both murine tissue explants and bone marrow-derived dendritic cells (data not shown). Furthermore, i.p. injection of mice with IL-36 $\alpha$ also leads to a rapid influx of inflammatory macrophages and neutrophils into the peritoneal cavity (Supplementary Figure 3).

To extend these studies and carry out a deeper mechanistic analysis of the role of IL-36R signaling in intestinal inflammation, we also investigated the responses of il36r - / mice using the $C$. rodentium model of infectious colitis. Along with the confirmation of the deregulated innate immune responses observed in the DSS model, these investigations also revealed IL-36R signaling as playing an important role in the generation of mucosal T helper subset responses. Specifically, we observed that while Th17-type responses were elevated earlier (day 7), Th1 responses were diminished in the colons of $i l 36 r-I$ - mice during the later phases of infection (day 14). Although these effects may be, at least in part, secondary to the deregulated innate inflammatory responses observed, it is striking that while confirming that IL-36 family members can promote Th1 responses, we also found that IL-36 can suppress Th17 responses in vitro. These data add significantly to our current knowledge of the instructive role of different IL-1 family members in promoting Th responses. For example, it is established that while IL-1 can promote Th17 responses, IL-18 is a potent inducer of Th1 responses and IL-33 acts to enhance Th2 type responses. ${ }^{10}$ These data suggest that IL-36 family members can act directly to enhance Th1 responses, while also suppressing Th17 cell development. These effects observed on the differentiation of Th17 responses are in contrast to a report by Vigne et al., which indicated that IL-36 could increase IL-17A expression by $\mathrm{CD} 4^{+} \mathrm{T}$ cells when stimulated under nonpolarizing conditions (albeit far less potently than its effects on increasing IFN $\gamma$ expression). For this reason, we specifically examined IL-17A expression by Th17 cells differentiated in vitro using $\mathrm{CD} 4+\mathrm{T}$ cells purified from ill17a-egfp reporter transgenic mouse. ${ }^{36}$ This allowed us to evaluate cytokine expression on a per cell basis directly by flow cytometry without the need for subsequent restimulation. It is also interesting to note that both we and others have found that IL-36 can act as a profound costimulus early during $\mathrm{CD} 4{ }^{+}$T-cell activation leading to significantly enhanced IL-2 expression $\left({ }^{13}\right.$ and data not shown). Elevated IL-2 can actively promote the differentiation of Th1 responses as well as actively suppressing the development of Th17 responses and may provide the mechanism through which IL-36 signaling modulates these responses. ${ }^{36-38}$

As well as promoting Th1 responses, we also found that IL-36 can act as a potent inducer of both Th2 and Th9 differentiation by murine $\mathrm{CD} 4+\mathrm{T}$ cells in vitro (Supplementary Figure 1B). This is of particular relevance in the context of UC, where mucosal inflammation response has previously been described as having the characteristics of an 'atypical' type 2 and/or Th9 response. ${ }^{24,39,40}$ Indeed, we also observed that levels of IL13 gene expression were specifically elevated among UC patients in our pediatric patient cohort, while IL9 expression was not detected (Supplementary Figure 1). These data indicate that IL-36 family members may also play an important role in type 2- and Th9-dependent inflammation in the context of UC, which warrants further investigation.

Together these data demonstrate for the first time an important role for IL-36 cytokines in the pathogenesis of intestinal inflammation with particular relevance to UC. As such the IL-36 family may represent an important new therapeutic axis for intervention in IBD.

\section{METHODS}

Study subjects. All pediatric patients and control participants were recruited with consent under the Determinants and Outcomes of CHildren and AdolescentS with IBD study at the gastroenterology unit at Our Lady's Children's Hospital Crumlin under approval from the institutional Research Ethics Committee (GEN/193/11). All patients have undergone diagnostic evaluation according to international pediatric standards (Porto criteria) following which, each case was rigorously phenotyped using the pediatric-specific Paris classification of IBD. Established pediatric evidence-based indices are used to document clinical disease activity—a Physician Global Assessment score for each case; the Paediatric Crohn's Disease Activity Index (PCDAI) for CD and Paediatric Ulcerative Colitis Activity Index (PUCAI) for UC (see Supplementary Table 1). Rectal biopsies were obtained from patients with informed consent/assent as part of routine diagnostic evaluation upon initial enrollment in patients who were treatment-naive. Patients, who were initially enrolled with suspected IBD, but subsequently not diagnosed with disease, comprise the control population. For immunohistochemistry, tissue sections were obtained from the archives of Cork University Hospital from patients with active UC as diagnosed by conventional clinical, endoscopic and radiological criteria. The determination of disease activity was made at time of endoscopy and subsequently by histopathological analysis. Biopsies were obtained from healthy controls undergoing routine screening for colon cancer. The demographics of the study population are as follows: male:female: (UC) 5:4, (HL) 4:5. Median age (years) (range) (UC) 35 (19-62) and (HL) 49 (22-71).

Study approval. All human and animal studies were carried out with approval from institutional ethics committees at Our Lady's Children's Hospital Crumlin, Cork University Hospital, University College Cork and Trinity College Dublin. Written informed consent/assent was received from patients enrolled in the Determinants and Outcomes of CHildren and AdolescentS with IBD clinical study prior to their inclusion in the study. 
Mice. Il36r - / - mice were obtained from Amgen and bred in house on a C57BL/6 background. For all experiments wild-type (WT) or il36r $\mathrm{r}^{+1-}$ heterozygous littermate mice were used as controls. Il17a-egfp mice were described previously. ${ }^{36}$ All mice used were between 6-8 weeks and were housed under specific pathogen-free conditions at Institute for Molecular Medicine, St James Hospital Dublin. All animal experiments were performed under license from the Irish Health Products Regulatory Authority (Project No. AE19136/P036) and Irish Dept of Health (B100/4108) and approved by the institutional ethical review boards (151214) (2011/023).

Immunohistochemistry. Immunohistochemical staining was performed in 4 - $\mu$ m-thick formalin-fixed, paraffin-embedded wholesection slides obtained from the archives of Cork University Hospital ( $n=9$ per group). Sections were deparaffinized in xylene and rehydrated before analysis. Antigen retrieval was performed by microwave irradiation in $0.01 \mathrm{M}$ citrate buffer, $\mathrm{pH}$ 6.0. Slides were washed twice for $5 \mathrm{~min}$ in a wash buffer containing $50 \mathrm{mM}$ Tris-Cl, pH 7.6; $50 \mathrm{mM} \mathrm{NaCl}$; $0.001 \%$ saponin. Endogenous peroxidase was quenched with $3.0 \%$ hydrogen peroxide in methanol for $10 \mathrm{~min}$. Nonspecific binding was blocked using $5 \%$ serum in wash buffer for $1 \mathrm{~h}$. Sections were incubated overnight at $4{ }^{\circ} \mathrm{C}$ with primary antibody. Primary antibody binding was localized using a biotinylated secondary antibody, and visualized using avidin-conjugated horseradish peroxidase and 3,3'-diaminobenzidine (DAB) substrate, contained within the Vectastain $\mathrm{ABC}$ detection kit (Vector Laboratories, Burlingame, CA). Slides were counterstained with haematoxylin and mounted. The IL36 Receptor antibody was obtained from Abcam (ab180894, Cambridge, UK) and used at a dilution of 1:2,000. The IL-36 alpha antibody was also obtained from Abcam (ab180909) and used at a dilution of 1:1,000. Controls were treated as above omitting the primary antibodies and also by using an IgG control in place of primary antibody. Analysis of results was performed independently by a trained histopathologist.

Induction of DSS colitis. Both WT control and il36r $-/$ - mice were housed together for 2-3 weeks before commencing studies. Colitis was induced by adding $2 \%(\mathrm{w} / \mathrm{v})$ DSS to drinking water. Mice were monitored daily for up to 7 days. Weight loss, stool consistency and rectal bleeding were recorded and used to calculate Disease Activity Index. The score ranges from 0 to 12 , which represents the sum of scores for weight loss, stool consistency and rectal bleeding. For histology, tissue was fixed overnight in $10 \%$ formalin before dehydration and embedding into paraffin blocks. Sections were subsequently cut and stained with haematoxylin and eosin. Histological scoring was performed in a blinded fashion using a system described previously. ${ }^{41}$ MPO activity in colon tissue lysates was determined by a colorimetric assay (Sigma Aldrich, Wicklow, Ireland) and carried out according to manufacturer's instructions.

Citrobacter rodentium infection model. Il36r $+/$ - littermate control and $i l 36 r-/-$ mice were randomly divided in groups of 5-7 mice and orally gavaged with $0.2 \mathrm{ml}$ of the bioluminescent $C$. rodentium derivative strain ICC180 $\left(4 \times 10^{9}\right.$ colony forming units $)$ and monitored for clinical signs of disease and body weight changes as described. ${ }^{26,30}$ Control mice were gavaged with PBS. Fecal samples were collected at different timepoints and spleen and liver were collected at the time of euthanasia on days 14 post infection (p.i.) and enumerated for bacterial load as described previously. ${ }^{30}$ In vivo bioluminescence imaging of colons, cecum, liver, spleen and mesenteric lymph nodes was performed using the Xenogen-IVIS Lumina II (Caliper Life Sciences, Hopkinton, MA). Quantification of bioluminescent signals was performed on regions of interest lay over the same surface area of the dissected colon, cecum, spleen, liver and mesenteric lymph nodes per individual animal. Background luminescence signal was omitted using representative average background regions of interests of non-luminescence levels from each image and on the uninfected animals. Imaging data were analyzed and quantified with Living Image software (version 4.4; Caliper Life Sciences) and expressed as total flux ( $\mathrm{p} / \mathrm{s})$.
After imaging, the length and weight of the colon was measured. Of the distal $3 \mathrm{~cm}, 0.5 \mathrm{~cm}$ was kept in RNA later and frozen for quantitative PCR with reverse transcription analysis. The rest of the tissue were divided longitudinally and frozen in liquid nitrogen for protein expression/activity analysis.

RNA analysis. RNA extractions from all patient samples were carried out using Bioline's (London, UK) RNA extraction kit according to manufacturer's instructions. Equal amounts of RNA were reverse transcribed using Applied Biosystems (Foster City, CA) high capacity cDNA reverse transcription kit according to manufacturer's instructions. Real-time PCR was performed using SensiFAST Probe Hi-ROX kit (Bioline) incorporating predesigned gene expression assays for genes shown (Applied Biosystems). Expression was normalized to $18 \mathrm{~s}$ ribosomal RNA. Samples were assayed on an Applied Biosystems 7900HT Fast Real-Time PCR System.

Statistics. Data are presented as mean \pm s.e.m. unless otherwise stated. Statistical significance was determined with one-way analysis of variance with post hoc analysis or the unpaired students $t$-test as outlined in the figure legends. Real-time quantitative PCR expression data, following 2$\Delta \Delta \mathrm{Ct}$ analysis were subjected to the Mann-Whitney $t$-test. All statistical tests were performed using Prism6 software (GraphPad Software, La Jolla, CA). A $P$-value of 0.05 was considered significant.

SUPPLEMENTARY MATERIAL is linked to the online version of the paper at http://www.nature.com/mi

\section{ACKNOWLEDGMENTS}

We acknowledge the patients (and their parents) enrolled in the Determinants and Outcomes of CHildren and AdolescentS with IBD clinical study, David Coyle and Alan Wolfe for technical assistance. Jason Radford and colleagues at the Biological Service Unit UCC for their technical support with the C. rodentium study. Maureen O'Sullivan and Roger Preston and Sarah Doyle for critical review of the data and manuscript. This work is supported by Science Foundation Ireland (PIYRA award 10/YI/B1827 to PTW and SFI/12/RC/2273 to FS and the APC Microbiome Institute) and the National Childrens Research Centre to PTW (PRiTI programme) and SH.

\section{DISCLOSURE}

The authors declare no conflict of interest.

c 2016 Society for Mucosal Immunology

\section{REFERENCES}

1. Hibi, T., Inoue, N., Ogata, H. \& Naganuma, M. Introduction and overview: recent advances in the immunotherapy of inflammatory bowel disease. J. Gastroenterol. 38 (Suppl 15), 36-42 (2003).

2. Ogata, H. \& Hibi, T. Cytokine and anti-cytokine therapies for inflammatory bowel disease. Curr. Pharm. Des. 9, 1107-1113 (2003).

3. Billiet, T., Rutgeerts, P., Ferrante, M., Van Assche, G. \& Vermeire, S. Targeting TNF-alpha for the treatment of inflammatory bowel disease. Expert Opin. Biol. Ther. 14, 75-101 (2014).

4. Perrier, C. \& Rutgeerts, P. New drug therapies on the horizon for IBD. Dig. Dis. 30 (Suppl 1), 100-105 (2012).

5. Khor, B., Gardet, A. \& Xavier, R.J. Genetics and pathogenesis of inflammatory bowel disease. Nature 474, 307-317 (2011).

6. Hope, B. et al. Rapid rise in incidence of Irish paediatric inflammatory bowel disease. Arch. Dis. Child 97, 590-594 (2012).

7. Henderson, P. \& Wilson, D.C. The rising incidence of paediatric-onset inflammatory bowel disease. Arch. Dis. Child 97, 585-586 (2012).

8. Gresnigt, M.S. \& van de Veerdonk, F.L. Biology of IL-36 cytokines and their role in disease. Semin. Immunol. 25, 458-465 (2013).

9. Tripodi, D. et al. IL-36 a new member of the IL-1 family cytokines. J Biol Regul Homeost Agents 26, 7-14 (2012).

10. Garlanda, C., Dinarello, C.A. \& Mantovani, A. The interleukin-1 family: back to the future. Immunity 39, 1003-1018 (2013). 
11. Towne, J.E. et al. Interleukin-36 (IL-36) ligands require processing for full agonist (IL-36alpha, IL-36beta, and IL-36gamma) or antagonist (IL-36Ra) activity. J. Biol. Chem. 286, 42594-42602 (2011).

12. Vigne, S. et al. IL-36R ligands are potent regulators of dendritic and Tcells. Blood 118, 5813-5823 (2011).

13. Vigne, S. et al. IL-36 signaling amplifies Th1 responses by enhancing proliferation and Th1 polarization of naive CD4 + T cells. Blood 120, 3478-3487 (2012).

14. Marrakchi, S. et al. Interleukin-36-receptor antagonist deficiency and generalized pustular psoriasis. N. Engl. J. Med. 365, 620-628 (2011).

15. Tortola, L. et al. Psoriasiform dermatitis is driven by IL-36-mediated DCkeratinocyte crosstalk. J. Clin. Invest. 122, 3965-3976 (2012).

16. Blumberg, $\mathrm{H}$. et al. Opposing activities of two novel members of the IL-1 ligand family regulate skin inflammation. J. Exp. Med. 204, 2603-2614 (2007).

17. Ramadas, R.A., Ewart, S.L., Medoff, B.D. \& LeVine, A.M. Interleukin-1 family member 9 stimulates chemokine production and neutrophil influx in mouse lungs. Am. J. Respir. Cell Mol. Biol. 44, 134-145 (2011).

18. Ramadas, R.A., Ewart, S.L., Iwakura, Y., Medoff, B.D. \& LeVine, A.M. IL36alpha exerts pro-inflammatory effects in the lungs of mice. PLoS One 7 , e45784 (2012).

19. van de Veerdonk, F.L. et al. IL-38 binds to the IL-36 receptor and has biological effects on immune cells similar to IL-36 receptor antagonist. Proc. Natl Acad. Sci. USA 109, 3001-3005 (2012).

20. Gresnigt, M.S. et al. The IL-36 receptor pathway regulates Aspergillus fumigatus-induced Th1 and Th17 responses. Eur. J. Immunol. 43, 416426 (2013)

21. Daig, R. et al. Increased interleukin 8 expression in the colon mucosa of patients with inflammatory bowel disease. Gut 38, 216-222 (1996).

22. Geremia, A. et al. IL-23-responsive innate lymphoid cells are increased in inflammatory bowel disease. J. Exp. Med. 208, 1127-1133 (2011).

23. Neurath, M.F. Cytokines in inflammatory bowel disease. Nat. Rev. Immunol. 14, 329-342 (2014).

24. Heller, F. et al. Interleukin-13 is the key effector Th2 cytokine in ulcerative colitis that affects epithelial tight junctions, apoptosis, and cell restitution. Gastroenterology 129, 550-564 (2005).

25. Kim, Y.G. et al. The Nod2 sensor promotes intestinal pathogen eradication via the chemokine CCL2-dependent recruitment of inflammatory monocytes. Immunity 34, 769-780 (2011).

26. Yang, S. et al. Pellino3 ubiquitinates RIP2 and mediates Nod2-induced signaling and protective effects in colitis. Nat Immunol 14, 927-936 (2013).
27. Ghosn, E.E. et al. Two physically, functionally, and developmentally distinct peritoneal macrophage subsets. Proc. Natl Acad. Sci. USA 107, 2568-2573 (2010).

28. Gabay, C. \& Towne, J.E. Regulation and function of interleukin-36 cytokines in homeostasis and pathological conditions. J. Leukoc. Biol. 97, 645-652 (2015).

29. Atarashi, K. et al. Th17 cell induction by adhesion of microbes to intestinal epithelial cells. Cell 163, 367-380 (2015).

30. Murphy, C.T. et al. The sphingosine-1-phosphate analogue FTY720 impairs mucosal immunity and clearance of the enteric pathogen Citrobacter rodentium. Infect. Immun. 80, 2712-2723 (2012).

31. Hall, L.J. et al. Natural killer cells protect against mucosal and systemic infection with the enteric pathogen Citrobacter rodentium. Infect. Immun. 81, 460-469 (2013)

32. Collins, J.W. et al. Citrobacter rodentium: infection, inflammation and the microbiota. Nat. Rev. Microbiol. 12, 612-623 (2014).

33. Bernstein, C.N., Wajda, A. \& Blanchard, J.F. The clustering of other chronic inflammatory diseases in inflammatory bowel disease: a population-based study. Gastroenterology 129, 827-836 (2005).

34. Weng, X., Liu, L., Barcellos, L.F., Allison, J.E. \& Herrinton, L.J. Clustering of inflammatory bowel disease with immune mediated diseases among members of a northern California-managed care organization. Am. J. Gastroenterol. 102, 1429-1435 (2007).

35. Marzano, A.V., Borghi, A., Stadnicki, A., Crosti, C. \& Cugno, M. Cutaneous manifestations in patients with inflammatory bowel diseases: pathophysiology, clinical features, and therapy. Inflamm. Bowel Dis. 20, 213-227 (2014).

36. Russell, S.E., Moore, A.C., Fallon, P.G. \& Walsh, P.T. Soluble IL-2Ralpha (sCD25) exacerbates autoimmunity and enhances the development of Th17 responses in mice. PLoS One 7, e47748 (2012).

37. Laurence, A. et al. Interleukin-2 signaling via STAT5 constrains T helper 17 cell generation. Immunity 26, 371-381 (2007).

38. Liao, W., Lin, J.X. \& Leonard, W.J. Interleukin-2 at the crossroads of effector responses, tolerance, and immunotherapy. Immunity 38, 13-25 (2013).

39. Gerlach, K. et al. TH9 cells that express the transcription factor PU.1 drive T cell-mediated colitis via IL-9 receptor signaling in intestinal epithelial cells. Nat. Immunol. 15, 676-686 (2014).

40. Gerlach, K., McKenzie, A.N., Neurath, M.F. \& Weigmann, B. IL-9 regulates intestinal barrier function in experimental T cell-mediated colitis. Tissue Barriers 3, e983777 (2015).

41. Smith, P. et al. Infection with a helminth parasite prevents experimental colitis via a macrophage-mediated mechanism. J. Immunol. 178, 4557-4566 (2007). 